de tumeurs chez des patients atteints de myélome multiple en rechute ou réfractaire [2]. Ces données ont alors conduit à la réalisation de l'essai de phase IIb présenté ici évaluant le sélinexor, combiné à la dexaméthasone, chez des patients atteints de myélomes multiples triple réfractaires [3].

Entre mai 2015 et mars 2018, 123 patients atteints de myélomes multiples triple réfractaires ont été inclus dans cette étude. L'âge médian des patients était de 65 ans (40-86) et le délai médian depuis le diagnostic de six ans (1-23). Cinquantetrois pour cent des patients présentaient des anomalies cytogénétiques dites de haut risque - délétion $\mathrm{du} \quad 17 \mathrm{p}$ (del[17p]), translocation $(4 ; 14)(\mathrm{t}[4 ; 14])$ ou $\mathrm{t}(14 ; 16)$ - tandis que le nombre médian de lignes antérieures de traitement était de sept (3-18). Quatre-vingt-six patients $(70 \%)$ avaient reçu du daratumumab, 102 patients (84\%) une autogreffe de cellules souches hématopoïétiques et deux patients (2\%) un traitement antérieur par cellules $\mathrm{T}$ à récepteurs antigéniques chimériques (CAR-T). Au total, quatre-vingt-trois patients (68\%) présentaient un myélome multiple pentaréfractaire (bortézomib, carfilzomib, lénalidomide, pomalidomide et daratumumab).
Suite à l'initiation de la combinaison sélinexor-dexaméthasone, 118 patients (96\%) ont interrompu le traitement, principalement en raison d'un effet indésirable grave ou de la progression de la pathologie, et la durée médiane de traitement était de neuf semaines (1-60). Les principaux effets indésirables de grade $\geq 3$ étaient hématologiques, incluant des thrombopénies (59\%), des anémies (44\%) et des neutropénies (21\%). Par ailleurs, $22 \%$ des patients présentaient des hyponatrémies de grade $\geq 3$. Ces effets indésirables graves, imputables au sélinexor ou à la dexaméthasone, ont conduit à l'interruption du traitement chez $18 \%$ des patients ; et des modifications de dose, voire des arrêts de traitement, furent nécessaires pour $80 \%$ des patients.

En termes d'efficacité, le taux de réponse globale était de $26 \%$ (95\% CI : 19-35), avec respectivement 20, 5 et $2 \%$ des patients en réponse partielle, en réponse partielle de très bonne qualité et en réponse complète stringente. Le délai médian de réponse était quatre mois (1-14) et la durée médiane de réponse de quatre mois (4-11). Les médianes de survie sans progression et globale étaient respectivement de quatre mois (95\%CI : 3-5) et neuf mois (95\%CI : 6-11). Chez les patients ayant présenté au moins une réponse minimale, la médiane de survie globale atteignait seize mois (95\% CI : 13-NA). Enfin, la quantification des ARNm d'XPO1 et de quatre protéines régulatrices (IRF3, ARL2BP, ZBTB17 et ATRX) ne permettait pas de distinguer les patients répondeurs au traitement.

En conclusion, cette étude de phase IIb démontre l'efficacité et la faisabilité d'un traitement par sélinexordexaméthasone chez les patients atteints de myélomes multiples triple réfractaires. Cette combinaison innovante permet l'obtention d'une réponse chez $26 \%$ de ces patients lourdement prétraités, de haut risque et en majorité pentaréfractaires. Bien que les effets indésirables hématologiques de grade $\geq 3$ soient fréquents, la progression rapide de ces patients et l'absence d'alternative thérapeutique rendent ce profil de toxicité acceptable. ]

\section{Références}

[1] Kumar SK, Dispenzieri A, Lacy MQ, et al. Continued improvement in survival in multiple myeloma: changes in early mortality and outcomes in older patients. Leukemia $2014 ; 28: 1122-8$.

[2] Vogl DT, Dingli D, Cornell RF, et al. Selective Inhibition of Nuclear Export With Oral Selinexor for Treatment of Relapsed or Refractory Multiple Myeloma. J Clin Oncol 2018 ; 36 : 859-66.

[3] Chari A, Vogl DT, Gavriatopoulou M, et al. Oral Selinexor-Dexamethasone for Triple-Class Refractory Multiple Myeloma. N Engl J Med 2019 ; 381 : 727-38.

\title{
Toxicité hématologique précoce et tardive des cellules $T$ à récepteurs antigéniques chimériques anti-CD19
}

\section{Jérôme Paillassa}

es cellules $\mathrm{T}$ à récepteurs antigéniques chimériques (CAR-T) anti-CD19 ont récemment montré leur remarquable efficacité dans les leucémies aiguës lymphoblastiques $B$ (LAL B), les lymphomes non hodgkiniens B (LNH B) et les leucémies lymphoïdes chroniques (LLC). Les principales toxicités sont le syndrome de relargage cytokinique (SRC), la toxicité neurologique centrale et l'hypogammaglobulinémie liée à l'hypoplasie B. Des neutropénies, thrombopénies et anémies ont également été décrites, dont certaines tardives par rapport à l'injection des CAR-T [1, 2]. Une étude israélienne, récemment publiée dans Bone
Marrow Transplantation, a exploré ces cytopénies précoces et tardives et a tenté d'en expliquer les mécanismes physiopathologiques [3].

Les données de cette étude sont issues d'un essai prospectif de phase Ib/II dans lequel trente-cinq patients (quatorze enfants et vingt et un adultes, dix-neuf LAL et seize LNH, au moins deux lignes de traitements anté- 
rieures, $37 \%$ ayant préalablement reçu une allogreffe) ont reçu des CAR$\mathrm{T}$ anti-CD19 (production locale, domaine de costimulation CD28, dose 1 à $1,5 \times 10^{6}$ CAR-T/kg) après une lymphodéplétion par cyclophosphamide $\left(900 \mathrm{mg} / \mathrm{m}^{2}\right.$ à $\left.\mathrm{J}-2\right)$ et fludarabine $\left(25 \mathrm{mg} / \mathrm{m}^{2}\right.$ par jour de J-4 à $\mathrm{J}-2$ ). Les patients répondeurs ont reçu une allogreffe deux mois après la réinjection des CAR-T.

Le taux de RC est de $69 \%$ et de RP de $14 \%$. Soixante-dix-sept pour cent des patients ont développé un SRC (14\% de grade 3-4). Respectivement 94,80 et $51 \%$ des patients ont eu une neutropénie, une thrombopénie et une anémie après la réinjection des CAR-T. En ne tenant compte que des vngt-neuf patients répondeurs, $97 \%$ ont eu une neutropénie (72 $\%$ sévère $<500 / \mathrm{mm}^{3}$ ), $86 \%$ une thrombopénie $(28 \%$ sévère $<50000 / \mathrm{mm}^{3}$ ) et $55 \%$ ont nécessité une transfusion érythrocytaire. Le délai médian de survenue de la neutropénie est de trois jours après la réinjection, zéro pour la thrombopénie. La durée médiane de la neutropénie est de 19,5 jours (rang 0-63), trente-deux jours pour la thrombopénie (rang 1-64). Le pic de transfusions érythrocytaires est à J4. De manière intéressante, $93 \%$ des répondeurs ont eu une toxicité hématologique tardive (au-delà de J21) : $76 \%$ une neutropénie (34\% sévère), $76 \%$ une thrombopénie (21\% sévère) et $17 \%$ ont nécessité une transfusion érythrocytaire tardive. Ces toxicités hématologiques tardives sont plus fréquentes chez les patients ayant eu un SRC de grade 3-4 et/ou une allogreffe $<1$ an avant les CAR-T. Ces cytopénies tardives ne sont associées à aucune infection sévère et aucune hémorragique majeure. Seuls deux patients avec une neutropénie tardive ont reçu du G-CSF. La majorité des patients ayant une toxicité hématologique prolongée a un profil de neutropénie et de thrombopénie biphasique: une première phase de cytopénies précoces, puis une reconstitution hématologique transitoire, suivie d'une seconde phase de cytopénies tardives (figure 1). De façon notable, les taux sériques de SDF-1 (pour stromalderived factor $-1=$ CXCL-12) sont corrélés aux valeurs absolues des neutrophiles au-delà de J28. Dans la littérature, des neutropénies tardives ont été corrélées à la reconstitution

\section{Figure 1}

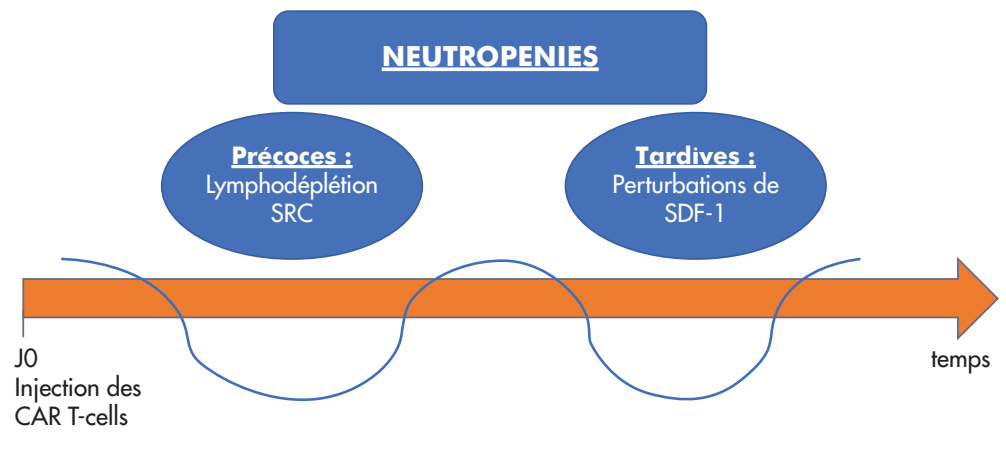

Profil biphasique de la toxicité hématologique post-CAR T-cells. SRC : syndrome de relargage cytokinique ; SDF-1 : stromal-derived factor-1. lymphocytaire B après un traitement par rituximab, avec un rôle causal des perturbations de SDF-1 [4].

Ainsi, si les toxicités hématologiques précoces post-injection de CAR-T sont liées à la lymphodéplétion et au syndrome de relargage cytokinique (avec des stigmates de syndrome d'activation macrophagique souvent associés), la neutropénie tardive semble liée à la consommation du SDF-1 par la reconstitution lymphocytaire B post-CAR-T (figure 1). En effet, SDF-1 est une chimiokine ayant un rôle à la fois dans la lymphopoïèse $B$ précoce, la migration des neutrophiles et la survie/migration des cellules souches hématopoïétiques. Une limite de cette étude reste toutefois l'absence de quantification de la reconstitution lymphocytaire $B$ après la réinjection des CAR-T, qui aurait permis de rechercher une corrélation entre cette reconstitution et la survenue d'une neutropénie tardive.

Les toxicités hématologiques après une réinjection de CAR-T anti-CD19 sont donc à la fois précoces et tardives, suivent une cinétique biphasique dans la majorité des cas, et ont des mécanismes physiopathologiques multiples. ]

\section{Références}

[1] Maude SL, Laetsch TW, Buechner J, et al Tisagenlecleucel in Children and Young Adults with B-Cell Lymphoblastic Leukemia. N Engl J Med 2018 ; $378: 439-48$.

[2] Cordeiro A, Bezerra ED, Hirayama AV, et al. Late Events after Treatment with CD19-Targeted Chimeric Antigen Receptor Modified T Cells. Biol Blood Marrow Transplant 2019. doi: 10.1016/j. bbmt.2019.08.003

[3] Fried S, Avigdor A, Bielorai B, et al. Early and late hematologic toxicity following CD19 CAR-T cells. Bone Marrow Transplant 2019; 54 1643-50

[4] Dunleavy K, Hakim F, Kim HK, et al. B-cell recovery following rituximab-based therapy is associated with perturbations in stromal derived factor-1 and granulocyte homeostasis. Blood $2005 ; 106$ : 795-802. 